\title{
Design, synthesis and biological evaluation of praziquantel and endoperoxide conjugates as antischistosomal agents
}

\begin{abstract}
Background: The widespread use of praziquantel for the treatment of schistosomiasis has led to concerns over the potential development of drug resistance. Therefore, the discovery of novel antischistosomal agents is imperative. In this study, a series of praziquantel and endoperoxide conjugates were synthesized and evaluated as potential antischistosomal agents. Results: Some compounds exhibited high efficacy against both adult and juvenile Schistosoma, in in vitro studies. Structureactivity relationship (SAR) analysis revealed that compounds with amide bond linker and cyclopentyl adjacent to the 1,2,4,5-tetraxane pharmacophore displayed the highest efficacy. Overall, compounds showed consistent activity against Schistosoma japonicum and Schistosoma mansoni. In vivo study resulted in moderate but statistically significant activity. Conclusion: Important preliminary results were obtained from thorough activity evaluation of praziquantel-endoperoxide conjugates. Further pharmacokinetic property investigation is necessary to improve in vivo efficacy.
\end{abstract}

Schistosomiasis is listed by WHO as one of the 17 th neglected tropical diseases (NTDs) [1]. The approximate global prevalence of schistosomiasis is 200 million and the annual death of this disease is about two hundred thousand. The disease is endemic in 76 countries worldwide, mainly in South America, southeast Asia and especially Africa, where infections are mediated through contact with cercariae released from infected snails in water. There are three main human pathogenic schistosome species, namely, Schistosoma mansoni, Schistosoma japonicum and Schistosoma haematobium.

Chemotherapy is the mainstay to fight against schistosome-acquired infections. After over 40 years of application, praziquantel (PZQ) (1, Figure 1) is still the only drug of choice for the treatment of schistosomiasis. This drug is highly effective against all three species of schistosomes at the adult stage, while barely efficient against the immature parasites $[2,3]$. Nonetheless, PZQ resistant or poorly sensitive strains have been identified in African countries [4,5] and resistant strain was also induced in the laboratory [6]. There are concerns that the widespread use of PZQ could potentially lead to emergence of resistant parasites in the near future. Hence, there is an urgent need for new drugs with novel mechanism of action.

Recently, there is an increasing interest in the discovery of agents that can concomitantly address more than one biological target for NTD treatment. It was believed that hybridization of two or more known pharmacophores would endow the new entity with functions from both selected templates [7]. For example, a hybrid compound named Trioxaquantel (2, Figure 1) and its derivatives were expected to possess antischistosomal activity [8]. A series of compounds named Trioxaquine (3, Figure 1) were designed and tested for their antimalarial and antischistosomal activity [9,10]. Conjugates bearing PZQ and synthetic endoperoxide pharmacophore were evaluated for antimalarial activity [11]. Organometallic derivatives of PZQ (4, Figure 1) were designed and evaluated as antischistosomal agents [12,13]. However, not all of these designed conjugates demonstrated expected biological activity.
Jing-jing Yang', Jerome Boissier', Jing-Lei Chen', Houzong Yao', Shilin Yang', Anne Rognon ${ }^{2} \&$ Chunhua Qiao*,1

Jiangsu Key Laboratory of Preventive \& Translational Medicine for Geriatric Diseases, College of Pharmaceutical Sciences, Soochow University 2Univ. Perpignan Via Domitia, IHPE UMR 5244, CNRS, IFREMER, Univ. Montpellier, F-66860 Perpignan, France

*Author for correspondence: qiaochunhua@suda.edu.cn 
<smiles>O=C(C1CCCCC1)N1CC(=O)N2CCc3ccccc3C2C1</smiles>

1. Praziquantel

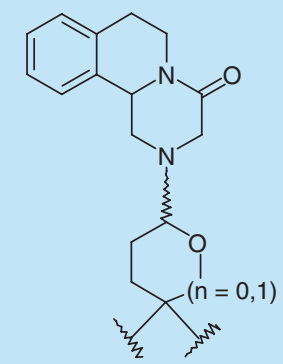

2. Trioxaquantels<smiles>CCC1(CC)CO[C@@H](Nc2ccnc3ccccc23)OO1</smiles>

3. Trioxaquines

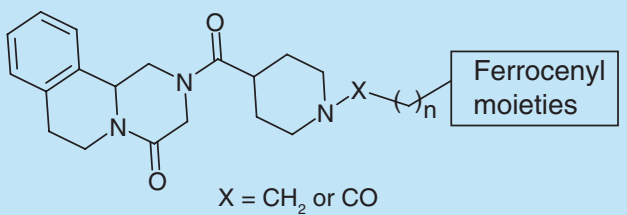

4. Organometal derivatives of praziquantel

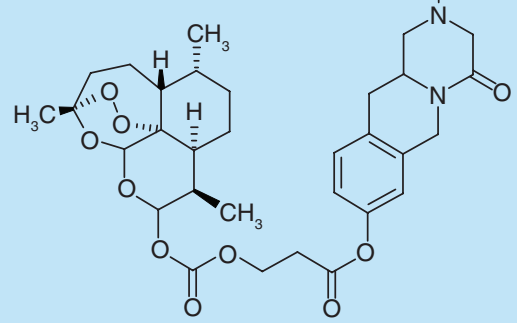

5. DW-3-15

Figure 1. Structures of (1) Praziquantel, (2) Trioxaquantels, (3) Trioxaquines, (4) Organometal derivatives of praziquantel and (5) DW-3-15.

This fact clearly emphasizes the need for a fundamentally different approach for the discovery of novel PZQ derivatives against schistosomiasis.

In the course of investigating PZQ derivatives as antischistosomal agents, we have reported DW-3-15 (5, Figure 1), an artesunate and PZQ hybrid, as a novel and promising prodrug with good efficacy against both adult and juvenile schistosomes [14,15]. However, DW-315 has a molecular weight $>700$ Dalton, which is much higher than the Lipinski defined MW500 for a druglike molecule. Moreover, the artemisinin derivative artesunate has limited natural resources. Low molecular weight surrogates of the artesunate endoperoxide pharmacophore obtainable by chemical preparation were extensively studied and reported. These structures include the 1,2,4,5-tetraoxane, 1,2,4-trioxolane and 1,2,4-trioxanes. Especially, as an effective antimalarial candidate, the synthetic compound OZ439 with 1,2,4-trioxolane pharmacophore offers new hope for a single-dose cure of uncomplicated malaria [16]. Inspired by these findings, we report here the design and synthesis of a series of endoperoxide and PZQ conjugates. We anticipated that they would have increased bioavailability, and thereby improved antischistosomal activity, by reducing the molecular weight. The antischistosomal activity of these compounds was evaluated both in vitro

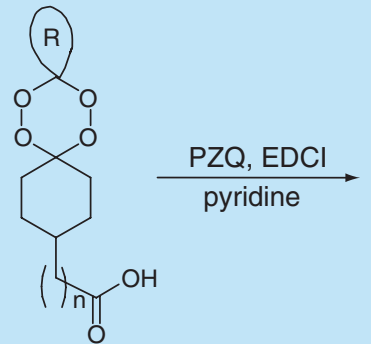

6a: $\mathrm{n}=0 ; \mathrm{R}=$ adamantyl 6b: $n=0 ; R=$ cyclopentyl 6c: $\mathrm{n}=0 ; \mathrm{R}=$ dimethyl $6 \mathrm{~d}: \mathrm{n}=1 ; \mathrm{R}=$ adamantyl 6e: $n=1 ; R=$ cyclohexyl 6f: $n=1 ; R=$ cyclopentyl $6 \mathrm{~g}: \mathrm{n}=1 ; \mathrm{R}=$ dimethyl

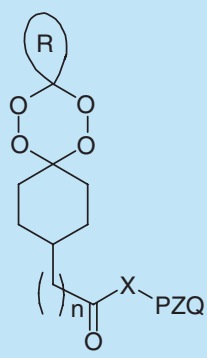

7: $\mathrm{n}=0 ; \mathrm{X}=\mathrm{NH} ; \mathrm{R}=$ adamantyl

8: $\mathrm{n}=0 ; \mathrm{X}=\mathrm{O} ; \mathrm{R}=$ adamantyl

9: $\mathrm{n}=0 ; \mathrm{X}=\mathrm{NH} ; \mathrm{R}=$ cyclopentyl

10: $\mathrm{n}=0 ; \mathrm{X}=\mathrm{O} ; \mathrm{R}=$ cyclopentyl

11: $\mathrm{n}=0 ; \mathrm{X}=\mathrm{NH} ; \mathrm{R}=$ dimethyl

12: $n=0 ; X=O ; R=$ dimethyl

13: $\mathrm{n}=1 ; \mathrm{X}=\mathrm{NH} ; \mathrm{R}=$ adamantyl

14: $\mathrm{n}=1 ; \mathrm{X}=\mathrm{O} ; \mathrm{R}=$ adamantyl

15: $n=1 ; X=N H ; R=$ cyclohexyl

16: $\mathrm{n}=1 ; \mathrm{X}=\mathrm{O} ; \mathrm{R}=$ cyclohexyl

17: $n=1 ; X=N H ; R=$ cyclopentyl

18: $n=1 ; X=O ; R=$ cyclopentyl

19: $\mathrm{n}=1 ; \mathrm{X}=\mathrm{NH} ; \mathrm{R}=$ dimethyl

20: $\mathrm{n}=1 ; \mathrm{X}=\mathrm{O} ; \mathrm{R}=$ dimethyl

Figure 2. Chemical synthesis route for compounds 7-20. 

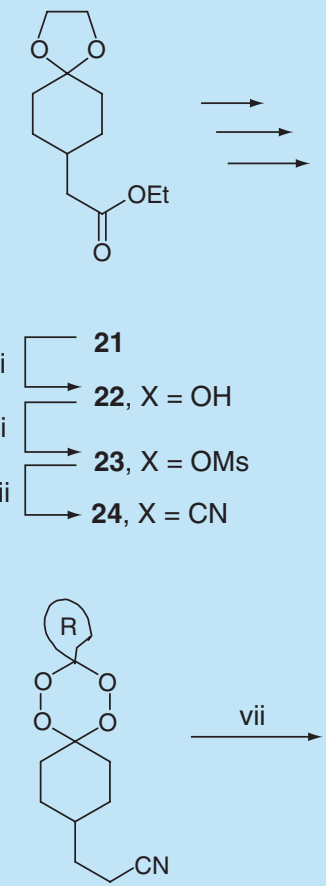

27a: $R=$ adamantyl

27b: $R=$ cyclohexyl

27c: $R=$ dimethyl

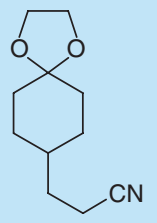

24

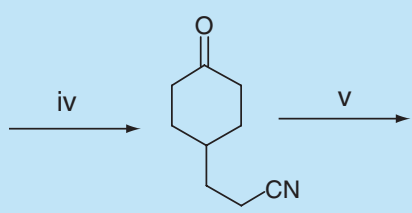

25

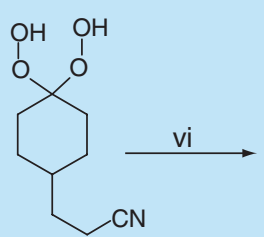

26

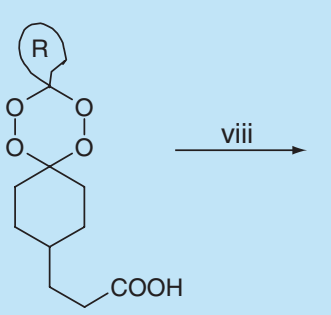

28a: $R$ = adamantyl

28b: $R=$ cyclohexyl

28c: $R=$ dimethyl

Figure 3. Chemical synthesis route for compounds 29-34.

and in vivo. A comparative study on in vitro activities in two parasite species, Schistosomamansoni and Schistosoma japonicum, was also conducted.

\section{Results \& discussion}

\section{Chemistry}

Reaction intermediates $\mathbf{6 a}, \mathbf{6 b}$ and $\mathbf{6 c}$ were prepared according to our previously described procedure [17]. Intermediates $\mathbf{6 d}, \mathbf{6 e}, \mathbf{6 f}$ and $\mathbf{6 g}$ were prepared according to a method reported by Amewu [18]. Condensation of the above carboxylic acids with 10-OH PZQ or $10-\mathrm{NH}_{2}$ PZQ [14] in the presence of coupling reagent EDCI afforded target compounds 7-20 (Figure 2).
Carboxylic acids 28a-c bearing the 1,2,4,5-tetraoxane moiety were synthesized in 7-step (Figure 2) starting from 21. Lithium aluminum hydride (LAH) reduction of the ester group gave the terminal alcohol 22 (90\%), which was followed by transformation of alcohol to mesylate (86\%) and subsequent substitution by sodium cyanide afforded cyanide $\mathbf{2 4}(80 \%)$, that was treated with $\mathrm{HCl}$ to provide the ketone 25 (55\%). The 1,1-dihydroperoxide derivative $\mathbf{2 6}$ was obtained by reaction of ketone with $30 \% \mathrm{H}_{2} \mathrm{O}_{2}$ in $40 \%$ yield. Reaction of $\mathbf{2 6}$ with the corresponding ketone provided 1,2,4,5-tetraoxanes 27a-c. Treatment of 27a-c with $\mathrm{NaOH}$ converted the cyanide group to carbox-

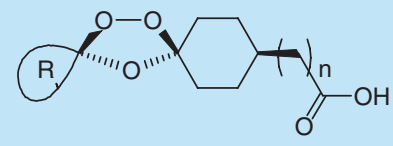

35a: $n=1: R=$ adamantyl 35b: $n=1 ; R=$ cyclohexyl

35c: $n=1 ; R=$ cyclopentyl

35d: $\mathrm{n}=2 ; \mathrm{R}=$ adamantyl

35e: $n=2 ; R=$ cyclohexyl
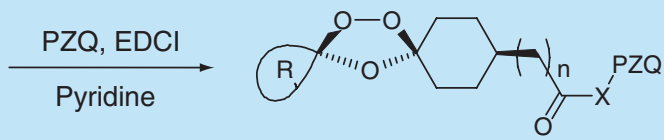

36: $\mathrm{n}=1 ; \mathrm{X}=\mathrm{NH} ; \mathrm{R}=$ adamantyl

37: $n=1 ; X=O ; R=$ adamantyl

38: $\mathrm{n}=1 ; \mathrm{X}=\mathrm{NH} ; \mathrm{R}=$ cyclohexyl

39: $\mathrm{n}=1 ; \mathrm{X}=\mathrm{O} ; \mathrm{R}=$ cyclohexyl

40: $\mathrm{n}=1 ; \mathrm{X}=\mathrm{NH} ; \mathrm{R}=$ cyclopentyl

41: $\mathrm{n}=1 ; \mathrm{X}=\mathrm{O} ; \mathrm{R}=$ cyclopentyl

42: $\mathrm{n}=2 ; \mathrm{X}=\mathrm{NH} ; \mathrm{R}=$ adamantyl

43: $\mathrm{n}=2 ; \mathrm{X}=\mathrm{O} ; \mathrm{R}=$ adamantyl

44: $\mathrm{n}=2 ; \mathrm{X}=\mathrm{NH} ; \mathrm{R}=$ cyclohexyl

45: $\mathrm{n}=2 ; \mathrm{X}=\mathrm{O} ; \mathrm{R}=$ cyclohexyl

Figure 4. Chemical synthesis route for compounds 36-45. 


\section{Key terms:}

Hybrid: Conjugate which is composed of and possesses functionality from two different pharmacophores.

Antischistosomal agent: Compound which can kill or shows efficacy against schistosoma.

Praziquantel derivatives: Compounds derived from praziquantel, or compounds containing the praziquantel moiety.

ylic acid to give $\mathbf{2 8 a}-\mathbf{c}$. Finally, coupling of $10-\mathrm{NH}_{2}$ or 10-OH PZQ with the above carboxylic acids afforded the synthetic target compounds 29-34 (Figure 3).

Synthesis conditions and reagents: LAH, THF, $0^{\circ} \mathrm{C}$; $\mathrm{MsCl}$, TEA, THF; KCN, DMSO, $120^{\circ} \mathrm{C}$; $\mathrm{HCl}$, actone; $30 \% \mathrm{H}_{2} \mathrm{O}_{2}$, conc. $\mathrm{HCl}, \mathrm{DCM}, \mathrm{CH}_{3} \mathrm{CN}$; ketone, PMA, $\mathrm{MgSO}_{4}, \mathrm{DCM} ; \mathrm{NaOH}, \mathrm{EtOH} ; \mathrm{PZQ}$, EDCI, pyridine.

Ozonide acids 35a-e were prepared using the method reported by Jonathan [19]. Compounds 3645 were prepared using the above mentioned EDCI coupling method (Figure 4).

\section{In vitro evaluation of compound activity} Activity against $\mathrm{S}$. japonicum in vitro

All prepared compounds were evaluated for their in vitro ability against both adult and juvenile Schistosoma japonicum according to a previously described method [20]. The results of repeated experiments against adult worm are shown in Table 1.

As shown in Table 1, most compounds displayed efficient worm killing ability. Specifically, compounds $9,11,12,15,17,19,31,33$ and 44 exhibited better potency than positive control PZQ: these compounds killed all worms within $72 \mathrm{~h}$ at concentrations lower than $25 \mu \mathrm{M}$, and several compounds displayed good efficacy at $5 \mu \mathrm{M}$. The activity of 7 , 20,34, 38, 40 and 45 was comparable to that of PZQ: at $25 \mu \mathrm{M}$, these compounds reduced the worm mobility score to $<20 \%$. The rest of the compounds displayed weaker worm killing capability. Overall, male worms were more vulnerable to these hybrid compounds.

In terms of structure activity relationship, several observations were concluded: compounds with amide bond linker displayed higher potency than compounds with ester linker ( 9 vs 10,15 vs 16,17 vs 18,19 vs 20,31 vs 34,38 vs 39,40 vs 41 ); the inhibitory activity of compounds with the $\mathrm{CH}_{2} \mathrm{CH}_{2}$ linker displayed low potency compared with those with shorter linkers $(15$ vs 30,16 vs 33,19 vs 31,20 vs 34,36 vs 42 and 37 vs 43 ); compounds bearing the cyclopentyl moiety exhibited higher inhibitory potency compared with those with cyclohexyl, adamantyl and isopropyl moieties (40 vs 36 and 38 ; 41 vs 37 and $39 ; 9$ vs 11 ; 17 vs 19,15 and $13 ; 18$ vs 20,16 and 14 ); compounds with the 1,2,4,5-tetraoxane pharmacophore exhibited higher worm killing ability compared with compounds with 1,2,4-trioxane pharmacophore (38 vs $\mathbf{1 5}, \mathbf{3 9}$ vs $16,40$ vs 17$)$.

Assessment of compound activity against juvenile worm in vitro

The inhibitory effect of these compounds against juvenile Schistosoma japonicum in vitro was also evaluated, and the result was displayed in Table 2.

Based on the above data, it was concluded that the compounds exhibited weak activity against juvenile worm compared with the adult worm, suggesting that the adult worms were more sensitive to these compounds in vitro. In general, compounds showed consistent activity against juvenile and the adult worm. Notably, compound $\mathbf{1 7}$ exhibited the most potent worm killing ability against the juvenile worms.

In order to infer the activity spectrum of these PZQendoperoxide conjugates, we have evaluated in vitro the activities of these compounds against adult Schistosoma mansoni as well (Table 3).

As shown in Table 3, the compounds can be classified into three categories: compounds killing all worms within 24 h (compounds 8, 9, 10, 11, 12, 17, 31, 34, 45), compounds killing all worms within $72 \mathrm{~h}$ (compounds 19, 30, 33) and poorly efficient compounds with worms remaining alive $72 \mathrm{~h}$ post treatment. Interestingly, among the nine most efficient compounds for the two parasite species, five of them (compounds 9, $11,12,17,31$ ) are common.

In vivo efficacy evaluation in infected mice model The effects of single $400 \mathrm{mg} / \mathrm{kg}$ oral dose of selected conjugates on worm burden reductions in 35-day-old adult and 21-day-old juvenile $S$. japonicum harbored in mice were evaluated, and the results are summarized in Table 4 and Table 5, respectively. Although conjugates 9, 16, 19 and 44 showed high efficacy against the adult worm in vitro, these compounds had no or low effect in vivo. Conjugates 11, 15, 17 and 31 showed $>20 \%$, but $<35 \%$, of worm killing activity against adult $S$. japonicum. Conjugates $\mathbf{1 7}$ and $\mathbf{1 9}$ had no statistically significant worm reduction effect against the juvenile worm. By comparison, conjugates 7, 9 and $\mathbf{1 1}$ showed significant activities on juvenile $S$. japonicum.

Overall, the in vivo activity of all conjugates was lower than that of the art-PZQ conjugate DW-3-15 [15]. The high in vitro activity was not transformed into high in vivo worm killing activity. This suggests that either these conjugates have a poor oral bioavailability, or different drug administration mode should be pursued 


\begin{tabular}{|c|c|c|c|c|c|c|c|c|}
\hline \multirow{2}{*}{$\begin{array}{l}\text { Compound } \\
\text { (No.) }\end{array}$} & \multirow{2}{*}{$\begin{array}{l}\text { Concentration } \\
(\mu \mathrm{M})\end{array}$} & \multirow{2}{*}{ Worm $^{+}$} & \multicolumn{2}{|r|}{$24 \mathrm{~h}$} & \multicolumn{2}{|c|}{$48 \mathrm{~h}$} & \multicolumn{2}{|c|}{$72 \mathrm{~h}$} \\
\hline & & & $\begin{array}{l}\text { Mobile } \\
\text { worm (\%) }\end{array}$ & $\begin{array}{l}\text { Mobility score } \\
\text { (\%) }\end{array}$ & $\begin{array}{l}\text { Mobile worm } \\
(\%)\end{array}$ & $\begin{array}{l}\text { Mobility } \\
\text { score (\%) }\end{array}$ & $\begin{array}{l}\text { Mobile } \\
\text { worm (\%) }\end{array}$ & $\begin{array}{l}\text { Mobility } \\
\text { score (\%) }\end{array}$ \\
\hline Control & 50 & $\hat{0}$ & 100 & 100 & 100 & 100 & 100 & 100 \\
\hline DMSO & & o & 100 & 100 & 100 & 100 & 100 & 100 \\
\hline \multirow[t]{4}{*}{7} & $50^{\ddagger}$ & $\hat{\sigma}$ & 100 & 33.3 & 75.0 & 33.3 & 0 & 0 \\
\hline & & q & 100 & 66.7 & 25.0 & 8.3 & 0 & 0 \\
\hline & 25 & $\hat{0}$ & 100 & $50.0 \pm 10.0$ & $40.0 \pm 20.0$ & $13.3 \pm 13.3$ & 0 & 0 \\
\hline & & q & 100 & $66.7 \pm 23.0$ & 100 & $41.7 \pm 12.3$ & $25.0 \pm 25.0$ & $8.3 \pm 8.3$ \\
\hline \multirow[t]{2}{*}{8} & $50^{\ddagger}$ & $\hat{0}$ & 100 & 73.3 & 60.0 & 60.0 & 60.0 & 46.7 \\
\hline & & o & 75.0 & 25.0 & 75.0 & 25.0 & 25.0 & 8.3 \\
\hline \multirow[t]{6}{*}{9} & $25^{\ddagger}$ & o & 25.0 & 8.3 & 0 & 0 & 0 & 0 \\
\hline & & q & 0 & 0 & 0 & 0 & 0 & 0 \\
\hline & $10^{ \pm}$ & $\hat{0}$ & 100 & 50.0 & 0 & 0 & 0 & 0 \\
\hline & & o & 100 & 33.3 & 0 & 0 & 0 & 0 \\
\hline & 5 & $0^{\pi}$ & 100 & $66.7 \pm 13.3$ & 0 & 0 & 0 & 0 \\
\hline & & q & 100 & $33.3 \pm 33.3$ & 0 & 0 & 0 & 0 \\
\hline \multirow[t]{4}{*}{10} & $50^{\ddagger}$ & 0 & 80.0 & 26.7 & 0 & 0 & 0 & 0 \\
\hline & & q & 25.0 & 8.3 & 0 & 0 & 0 & 0 \\
\hline & 25 & $\hat{0}$ & 100 & $33.3 \pm 8.3$ & 100 & $33.3 \pm 8.3$ & 100 & $33.3 \pm 8.3$ \\
\hline & & ㅇ & 100 & $33.3 \pm 16.6$ & 100 & $\begin{array}{l}33.3 \\
\pm 16.6\end{array}$ & 100 & $33.3 \pm 16.6$ \\
\hline \multirow[t]{4}{*}{11} & 25 & $\hat{0}$ & 100 & $50.0 \pm 25.0$ & 0 & 0 & 0 & 0 \\
\hline & & q & 100 & $33.3 \pm 33.3$ & 0 & 0 & 0 & 0 \\
\hline & 10 & $\hat{0}$ & 100 & $58.3 \pm 15.3$ & $20.0 \pm 10.0$ & $6.7 \pm 3.3$ & 0 & 0 \\
\hline & & q & 100 & $60.0 \pm 20.0$ & $50.0 \pm 10.0$ & $16.7 \pm 3.3$ & 0 & 0 \\
\hline \multirow[t]{4}{*}{12} & 25 & $0^{\pi}$ & 100 & $33.3 \pm 33.3$ & 100 & $\begin{array}{l}33.3 \\
\pm 33.3\end{array}$ & 0 & 0 \\
\hline & & 우 & 100 & $33.3 \pm 33.3$ & 0 & 0 & 0 & 0 \\
\hline & 10 & $\hat{0}$ & 100 & $33.3 \pm 33.3$ & 100 & $33.3 \pm 6.7$ & 50 & $16.7 \pm 16.6$ \\
\hline & & q & 100 & $33.3 \pm 33.3$ & 100 & $33.3 \pm 6.7$ & 50 & $16.7 \pm 8.3$ \\
\hline \multirow[t]{2}{*}{13} & $50^{\ddagger}$ & o & 100 & 91.7 & 100 & 91.7 & 100 & 66.7 \\
\hline & & q & 100 & 66.7 & 100 & 66.7 & 100 & 33.3 \\
\hline \multirow[t]{2}{*}{14} & $50^{\ddagger}$ & 0 & 100 & 33.3 & 20.0 & 6.7 & 20.0 & 6.7 \\
\hline & & q & 100 & 66.7 & 50.0 & 16.7 & 100 & 33.3 \\
\hline \multirow[t]{4}{*}{15} & 25 & 0 & 100 & $33.3 \pm 8.3$ & 0 & 0 & 0 & 0 \\
\hline & & q & 100 & $33.3 \pm 8.3$ & 0 & 0 & 0 & 0 \\
\hline & 10 & $0^{\pi}$ & 100 & $66.7 \pm 16.7$ & 0 & 0 & 0 & 0 \\
\hline & & q & 100 & $33.3 \pm 6.7$ & 0 & 0 & 0 & 0 \\
\hline 16 & $50^{\ddagger}$ & $\hat{\sigma}$ & 0 & 0 & 0 & 0 & 0 & 0 \\
\hline
\end{tabular}


Preliminary Communication Yang, Boissier, Chen et al.

\section{Table 1. Effects of the compounds on adult Schistosoma japonicum in vitro (cont.).}

\begin{tabular}{|c|c|c|c|c|c|c|c|c|}
\hline \multirow{2}{*}{$\begin{array}{l}\text { Compound } \\
\text { (No.) }\end{array}$} & \multirow{2}{*}{$\begin{array}{l}\text { Concentration } \\
(\mu \mathrm{M})\end{array}$} & \multirow[t]{2}{*}{ Worm ${ }^{+}$} & \multicolumn{2}{|r|}{$24 \mathrm{~h}$} & \multicolumn{2}{|c|}{$48 \mathrm{~h}$} & \multicolumn{2}{|c|}{$72 \mathrm{~h}$} \\
\hline & & & $\begin{array}{l}\text { Mobile } \\
\text { worm (\%) }\end{array}$ & $\begin{array}{l}\text { Mobility score } \\
\text { (\%) }\end{array}$ & $\begin{array}{l}\text { Mobile worm } \\
(\%)\end{array}$ & $\begin{array}{l}\text { Mobility } \\
\text { score (\%) }\end{array}$ & $\begin{array}{l}\text { Mobile } \\
\text { worm (\%) }\end{array}$ & $\begin{array}{l}\text { Mobility } \\
\text { score (\%) }\end{array}$ \\
\hline & & q & 0 & 0 & 0 & 0 & 0 & 0 \\
\hline & 25 & o & 100 & $33.3 \pm 6.7$ & 100 & $33.3 \pm 6.7$ & $25.0 \pm 25.0$ & $8.3 \pm 8.3$ \\
\hline & & 우 & 50 & $33.3 \pm 6.7$ & 100 & $33.3 \pm 6.7$ & 0 & 0 \\
\hline & 10 & $0^{\lambda}$ & 100 & $33.3 \pm 33.3$ & $75.0 \pm 25.0$ & $25.0 \pm 5.0$ & $75.0 \pm 25.0$ & $25 \pm 22.1$ \\
\hline & & 우 & 100 & $16.7 \pm 6.7$ & 0 & 0 & 0 & 0 \\
\hline \multirow[t]{6}{*}{17} & 25 & $\sigma^{\lambda}$ & 25 & $8.3 \pm 8.3$ & 0 & 0 & 0 & 0 \\
\hline & & q & 50 & $33.3 \pm 33.3$ & $50.0 \pm 25.0$ & $16.7 \pm 16.7$ & 0 & 0 \\
\hline & 10 & $0^{\pi}$ & 75 & $25.0 \pm 25.0$ & 0 & 0 & 0 & 0 \\
\hline & & o & 25 & $8.3 \pm 8.3$ & 0 & 0 & 0 & 0 \\
\hline & 5 & o & 100 & $44.4 \pm 22.2$ & $66.7 \pm 33.3$ & $22.2 \pm 11.1$ & 0 & 0 \\
\hline & & q & 100 & $33.3 \pm 6.7$ & $50.0 \pm 25.0$ & $16.7 \pm 16.7$ & $25 \pm 0.0$ & $8.3 \pm 8.3$ \\
\hline \multirow[t]{4}{*}{18} & 50 & $\sigma^{\lambda}$ & $0 \ddagger$ & $0 \neq$ & $0 \ddagger$ & $0 \neq$ & $0 \ddagger$ & $0 \ddagger$ \\
\hline & & 우 & $0 \ddagger$ & $0 \neq$ & $0 \neq$ & $0 \neq$ & $0 \ddagger$ & $0 \ddagger$ \\
\hline & 25 & o & 100 & $33.3 \pm 16.7$ & 100 & $33.3 \pm 16.7$ & $25 \pm 0.0$ & $25 \pm 22.1$ \\
\hline & & o & 100 & $33.3 \pm 16.7$ & 100 & $33.3 \pm 16.7$ & $25 \pm 0.0$ & $25 \pm 8.3$ \\
\hline \multirow[t]{6}{*}{19} & 25 & $\sigma^{\lambda}$ & 100 & $33.3 \pm 16.7$ & 0 & 0 & 0 & 0 \\
\hline & & o & 100 & $33.3 \pm 16.7$ & $33.3 \pm 33.3$ & $11.1 \pm 6.7$ & 0 & 0 \\
\hline & 10 & $0^{\lambda}$ & $50.0 \pm 25.0$ & $16.7 \pm 16.7$ & 0 & 0 & 0 & 0 \\
\hline & & o & 0 & 0 & 0 & 0 & 0 & 0 \\
\hline & 5 & $0^{\pi}$ & 100 & $66.7 \pm 33.3$ & 100 & $\begin{array}{l}66.7 \\
\pm 33.3\end{array}$ & 100 & $41.6 \pm 14.4$ \\
\hline & & q & 100 & $33.3 \pm 33.3$ & $50.0 \pm 25.0$ & $16.7 \pm 16.7$ & $25.0 \pm 25.0$ & $8.3 \pm 8.3$ \\
\hline \multirow[t]{4}{*}{20} & $50^{\ddagger}$ & $0^{\lambda}$ & 0 & 0 & 0 & 0 & 0 & 0 \\
\hline & & q & 0 & 0 & 0 & 0 & 0 & 0 \\
\hline & 25 & $\hat{0}$ & 100 & $33.3 \pm 16.7$ & 100 & $\begin{array}{l}33.3 \pm \\
16.7\end{array}$ & $50.0 \pm 0.0$ & $16.7 \pm 22.1$ \\
\hline & & 우 & 100 & $33.3 \pm 16.7$ & $75.0 \pm 25.0$ & $\begin{array}{l}25.0 \pm \\
22.1\end{array}$ & $75.0 \pm 25.0$ & $25.0 \pm 22.1$ \\
\hline \multirow[t]{2}{*}{29} & $50^{\ddagger}$ & $\sigma^{\lambda}$ & 100 & 100 & 100 & 100 & 100 & 58.3 \\
\hline & & q & 100 & 100 & 100 & 93.3 & 100 & 53.3 \\
\hline \multirow[t]{6}{*}{32} & $50^{\ddagger}$ & $\hat{0}$ & 0 & 0 & 0 & 0 & 0 & 0 \\
\hline & & o & 0 & 0 & 0 & 0 & 0 & 0 \\
\hline & 25 & $\hat{0}$ & 100 & $33.3 \pm 33.3$ & 100 & $\begin{array}{l}33.3 \\
\pm 33.3\end{array}$ & $25 \pm 25$ & $8.3 \pm 0$ \\
\hline & & of & 100 & $33.3 \pm 33.3$ & $50.0 \pm 33.3$ & $16.7 \pm 16.7$ & 0 & 0 \\
\hline & 10 & o & 100 & $33.3 \pm 33.3$ & 100 & $\begin{array}{l}33.3 \\
\pm 33.3\end{array}$ & 100 & $33.3 \pm 8.3$ \\
\hline & & o & 100 & $66.7 \pm 8.3$ & 100 & $66.7 \pm 8.3$ & 100 & $66.7 \pm 8.3$ \\
\hline
\end{tabular}




\begin{tabular}{|c|c|c|c|c|c|c|c|c|}
\hline \multirow{2}{*}{$\begin{array}{l}\text { Compound } \\
\text { (No.) }\end{array}$} & \multirow{2}{*}{$\begin{array}{l}\text { Concentration } \\
(\mu \mathrm{M})\end{array}$} & \multirow[t]{2}{*}{ Worm $^{+}$} & \multicolumn{2}{|r|}{$24 \mathrm{~h}$} & \multicolumn{2}{|c|}{$48 \mathrm{~h}$} & \multicolumn{2}{|c|}{$72 \mathrm{~h}$} \\
\hline & & & $\begin{array}{l}\text { Mobile } \\
\text { worm (\%) }\end{array}$ & $\begin{array}{l}\text { Mobility score } \\
(\%)\end{array}$ & $\begin{array}{l}\text { Mobile worm } \\
\text { (\%) }\end{array}$ & $\begin{array}{l}\text { Mobility } \\
\text { score (\%) }\end{array}$ & $\begin{array}{l}\text { Mobile } \\
\text { worm (\%) }\end{array}$ & $\begin{array}{l}\text { Mobility } \\
\text { score (\%) }\end{array}$ \\
\hline \multirow[t]{4}{*}{31} & 25 & o & 100 & $41.7 \pm 8.3$ & 0 & 0 & 0 & 0 \\
\hline & & ㅇ & 100 & $33.3 \pm 6.7$ & 0 & 0 & 0 & 0 \\
\hline & 10 & $\sigma^{\lambda}$ & 100 & $50.0 \pm 25.0$ & $50.0 \pm 25.0$ & $\begin{array}{l}25.0 \\
\pm 12.5\end{array}$ & 0 & 0 \\
\hline & & q & $75.0 \pm 25.0$ & $25.0 \pm 25.0$ & 0 & 0 & 0 & 0 \\
\hline \multirow[t]{2}{*}{30} & $50^{\ddagger}$ & ๙ & 100 & 33.3 & 100 & 33.3 & 100 & 33.3 \\
\hline & & 우 & 100 & 33.3 & 100 & 33.3 & 100 & 33.3 \\
\hline \multirow[t]{6}{*}{33} & $50^{\ddagger}$ & o & 0 & 0 & 0 & 0 & 0 & 0 \\
\hline & & 우 & 0 & 0 & 0 & 0 & 0 & 0 \\
\hline & 25 & o & $50.0 \pm 25.0$ & $16.7 \pm 16.7$ & 0 & 0 & 0 & 0 \\
\hline & & q & $25.0 \pm 25.0$ & $8.3 \pm 8.3$ & 0 & 0 & 0 & 0 \\
\hline & 10 & ๙ & 100 & $66.7 \pm 33.3$ & 100 & $\begin{array}{l}55.6 \\
\pm 10.4\end{array}$ & $33.3 \pm 33.3$ & $22.2 \pm 22.2$ \\
\hline & & q & 100 & $66.7 \pm 33.3$ & $40.0 \pm 20.0$ & $\begin{array}{l}13.3 \\
\pm 13.3\end{array}$ & $40.0 \pm 20.0$ & $13.3 \pm 13.3$ \\
\hline \multirow[t]{4}{*}{34} & $50^{\ddagger}$ & त & 100 & 33.3 & 100 & 33.3 & 25 & 8.3 \\
\hline & & 우 & 100 & 33.3 & 0 & 0 & 0 & 0 \\
\hline & 25 & ๙ & 100 & $33.3 \pm 0.0$ & 100 & $33.3 \pm 0.0$ & $75.0 \pm 25.0$ & $25.0 \pm 22.1$ \\
\hline & & 우 & 100 & $33.3 \pm 0.0$ & $50.0 \pm 25.0$ & $16.7 \pm 16.7$ & $25.0 \pm 0.0$ & $8.3 \pm 8.3$ \\
\hline \multirow[t]{4}{*}{36} & $50^{\ddagger}$ & $\sigma^{\pi}$ & 100 & 66.7 & 100 & 33.3 & 25.0 & 8.3 \\
\hline & & o & 100 & 66.7 & 100 & 66.7 & 50.0 & 16.7 \\
\hline & 25 & $0^{\pi}$ & 100 & $77.5 \pm 2.5$ & $83.3 \pm 16.7$ & $44.4 \pm 11.1$ & $66.7 \pm 33.3$ & $33.3 \pm 8.3$ \\
\hline & & ㅇ & 100 & $66.7 \pm 16.7$ & 100 & $33.3 \pm 8.3$ & 100 & $33.3 \pm 8.3$ \\
\hline \multirow[t]{4}{*}{37} & $50^{\ddagger}$ & ๙ & 40.0 & 15.3 & 0 & 0 & 0 & 0 \\
\hline & & 우 & 100 & 46.7 & 0 & 0 & 0 & 0 \\
\hline & 25 & $0^{\lambda}$ & 100 & $66.7 \pm 33.3$ & 100 & $50.0 \pm 12.5$ & 100 & $33.3 \pm 8.3$ \\
\hline & & o & 100 & $33.3 \pm 8.3$ & 100 & $33.3 \pm 8.3$ & 100 & $33.3 \pm 8.3$ \\
\hline \multirow[t]{4}{*}{38} & $50^{\ddagger}$ & $0^{\pi}$ & 0 & 0 & 0 & 0 & 0 & 0 \\
\hline & & ㅇ & 0 & 0 & 0 & 0 & 0 & 0 \\
\hline & 25 & त & 100 & $66.7 \pm 33.3$ & $66.7 \pm 33.3$ & $\begin{array}{l}22.2 \pm \\
11.1\end{array}$ & $33.3 \pm 33.3$ & $11.1 \pm 11.1$ \\
\hline & & ㅇ & 100 & $50.0 \pm 25.0$ & 100 & $\begin{array}{l}33.3 \pm \\
33.3\end{array}$ & $50.0 \pm 25.0$ & $16.7 \pm 22.1$ \\
\hline \multirow[t]{4}{*}{39} & $50^{\ddagger}$ & $0^{\pi}$ & 0 & 0 & 0 & 0 & 0 & 0 \\
\hline & & q & 25 & 8.3 & 0 & 0 & 0 & 0 \\
\hline & 25 & $\sigma^{\pi}$ & 100 & $83.3 \pm$ & 100 & $41.7 \pm$ & 100 & $33.3 \pm 8.3$ \\
\hline & & 우 & 100 & $66.7 \pm$ & 100 & $66.7 \pm$ & 100 & $58.3 \pm 22.1$ \\
\hline 40 & $50^{\ddagger}$ & $0^{\pi}$ & 75.0 & 58.3 & 0 & 0 & 0 & 0 \\
\hline
\end{tabular}


Preliminary Communication Yang, Boissier, Chen et al.

Table 1. Effects of the compounds on adult Schistosoma japonicum in vitro (cont.).

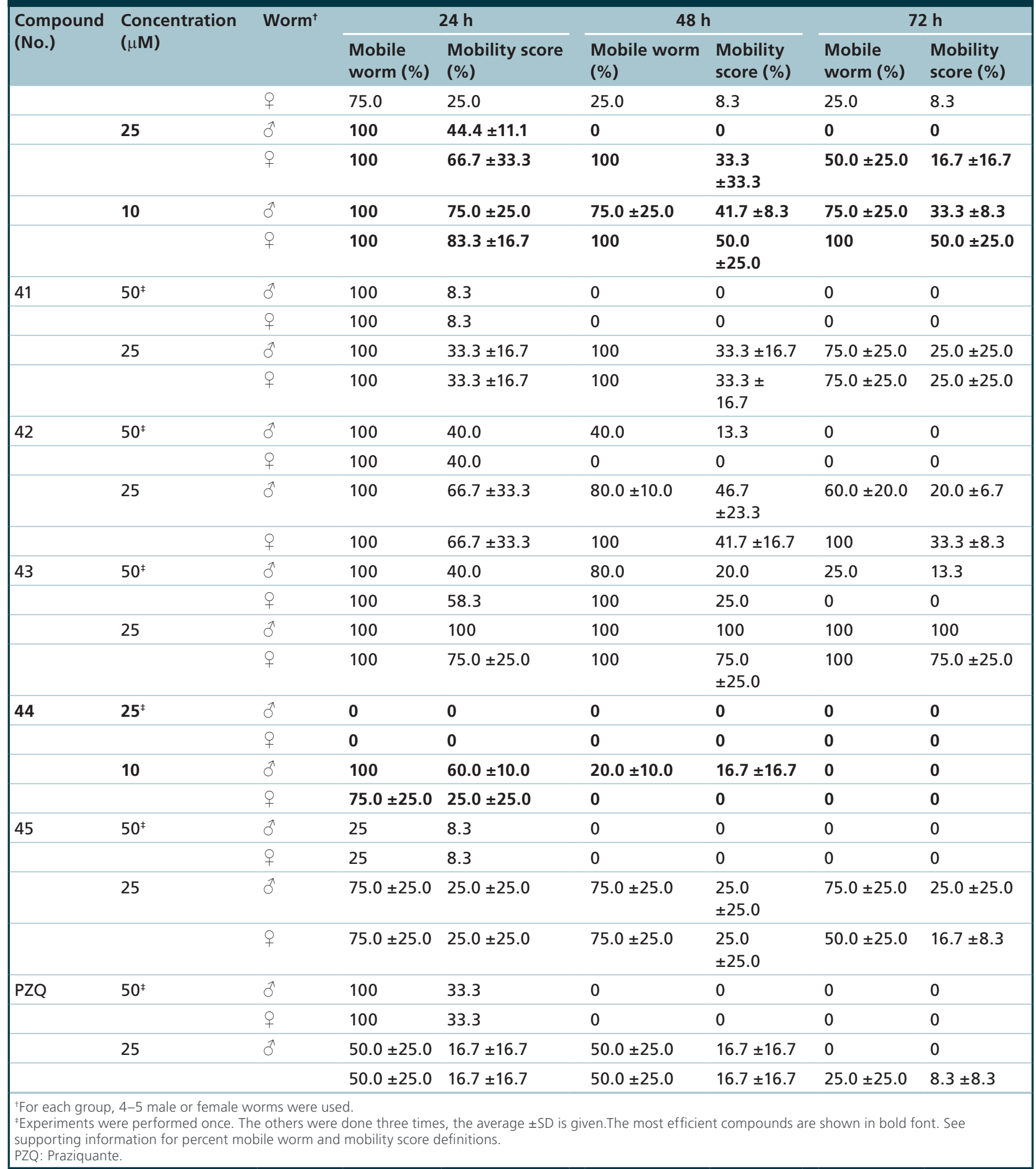




\begin{tabular}{|c|c|c|c|c|c|c|c|}
\hline \multirow{2}{*}{$\begin{array}{l}\text { Compound } \\
\text { (No.) }\end{array}$} & \multirow{2}{*}{$\begin{array}{l}\text { Concentration } \\
(\mu \mathrm{M})\end{array}$} & \multicolumn{2}{|c|}{$24 \mathrm{~h}$} & \multicolumn{2}{|c|}{$48 \mathrm{~h}$} & \multicolumn{2}{|c|}{$72 \mathrm{~h}$} \\
\hline & & $\begin{array}{l}\text { Mobile } \\
\text { worm (\%) }\end{array}$ & $\begin{array}{l}\text { Mobility } \\
\text { score (\%) }\end{array}$ & $\begin{array}{l}\text { Mobile } \\
\text { worm (\%) }\end{array}$ & $\begin{array}{l}\text { Mobility } \\
\text { score (\%) }\end{array}$ & $\begin{array}{l}\text { Mobile } \\
\text { worm (\%) }\end{array}$ & $\begin{array}{l}\text { Mobility } \\
\text { score (\%) }\end{array}$ \\
\hline Control & & 100 & 100 & 100 & 100 & 100 & 100 \\
\hline \multirow[t]{3}{*}{7} & $50^{+}$ & 100 & 55.6 & 33.3 & 11.1 & 0 & 0 \\
\hline & 25 & 100 & $50.0 \pm 25.0$ & $50.0 \pm 10.0$ & $16.7 \pm 8.4$ & $16.7 \pm 16.7$ & $5.6 \pm 5.6$ \\
\hline & 10 & 100 & $33.3 \pm 16.5$ & 100 & $33.3 \pm 6.5$ & $40 \pm 20$ & $13.3 \pm 6.7$ \\
\hline 8 & $50^{+}$ & 100 & 33.3 & 100 & 33.3 & 100 & 33.3 \\
\hline \multirow[t]{3}{*}{9} & $50^{+}$ & 55.6 & 27.8 & 0 & 0 & 0 & 0 \\
\hline & 25 & 22.2 & $7.4 \pm 3.5$ & $22.4 \pm 7.5$ & $7.4 \pm 3.8$ & $5.6 \pm 5.6$ & $1.9 \pm 1.9$ \\
\hline & 10 & 100 & $63.3 \pm 10.3$ & $84.6 \pm 4.5$ & $48.7 \pm 24.3$ & $50 \pm 10$ & $16.7 \pm 3.3$ \\
\hline 10 & 50 & $100^{+}$ & $58.3^{\dagger}$ & $100^{+}$ & $33.3^{+}$ & $100^{+}$ & $33.3^{+}$ \\
\hline \multirow[t]{3}{*}{11} & $50^{+}$ & 37.5 & 12.5 & 0 & 0 & 0 & 0 \\
\hline & 25 & 100 & $66.7 \pm 11.1$ & 100 & $55.6 \pm 24.5$ & $66.7 \pm 11.1$ & $33.3 \pm 3.7$ \\
\hline & 10 & 100 & $52.4 \pm 26.2$ & 100 & $52.4 \pm 26.2$ & 100 & $\begin{array}{l}52.4 \\
\pm 14.2\end{array}$ \\
\hline 12 & $50^{+}$ & 100 & 33.3 & 100 & 33.3 & 100 & 33.3 \\
\hline 13 & $50^{+}$ & 100 & 100 & 100 & 100 & 100 & 100 \\
\hline 14 & $50^{+}$ & 100 & 66.7 & 100 & 66.7 & 100 & 55.6 \\
\hline 15 & $50^{+}$ & 83.3 & 55.6 & 50.0 & 22.2 & 50.0 & 22.2 \\
\hline 16 & $50^{+}$ & 100 & 33.3 & 100 & 33.3 & 100 & 33.3 \\
\hline \multirow[t]{3}{*}{17} & $50^{+}$ & 37.5 & 12.5 & 0 & 0 & 0 & 0 \\
\hline & 25 & 50 & $36.7 \pm 3.5$ & $50.0 \pm 10.0$ & $20.0 \pm 5.0$ & $10.0 \pm 10.0$ & $3.3 \pm 6.6$ \\
\hline & 10 & 100 & $48.5 \pm 10.2$ & $54.5 \pm 27.2$ & $18.2 \pm 4.3$ & 0 & 0 \\
\hline \multirow[t]{3}{*}{18} & $50^{+}$ & 0 & 0 & 0 & 0 & 0 & 0 \\
\hline & 25 & $56.3 \pm 10.3$ & $33.3 \pm 12.3$ & $56.3 \pm 18.7$ & $31.1 \pm 12.3$ & $50.0 \pm 6.7$ & $22.2 \pm 6.7$ \\
\hline & 10 & 100 & $33.3 \pm 14.5$ & 100 & $33.3 \pm 13.5$ & $84.0 \pm 8.0$ & $28.0 \pm 4.0$ \\
\hline \multirow[t]{3}{*}{19} & $50^{+}$ & 57.1 & 19 & 0 & 0 & 0 & 0 \\
\hline & 25 & 100 & $66.7 \pm 11.1$ & $72.7 \pm 28.3$ & $39.4 \pm 32.4$ & $36.4 \pm 18.2$ & $21.2 \pm 9.1$ \\
\hline & 10 & 100 & $66.7 \pm 22.2$ & 100 & $60.0 \pm 10.0$ & $60.0 \pm 20.0$ & $33.3 \pm 6.7$ \\
\hline \multirow[t]{3}{*}{20} & $50^{+}$ & 0 & 0 & 0 & 0 & 0 & 0 \\
\hline & 25 & 100 & $55.6 \pm 15.6$ & 100 & $44.4 \pm 12.6$ & 100 & $44.4 \pm 5.6$ \\
\hline & 10 & 100 & $66.7 \pm 12.3$ & 100 & $66.7 \pm 12.3$ & $100 \pm$ & $66.7 \pm 4.8$ \\
\hline 29 & $50^{+}$ & 100 & 100 & 100 & 100 & 100 & 66.7 \\
\hline 30 & $50^{+}$ & 100 & 33.3 & 100 & 33.3 & 100 & 33.3 \\
\hline \multirow[t]{3}{*}{31} & $50^{+}$ & 60.0 & 40.0 & 40.0 & 20.0 & 0 & 0 \\
\hline & 25 & $84.6 \pm 5.2$ & $71.8 \pm 10.5$ & $46.2 \pm 21.3$ & $17.9 \pm 25.6$ & $30.8 \pm 15.4$ & $10.8 \pm 7.7$ \\
\hline & 10 & 100 & $90.5 \pm 3.2$ & 100 & $71.4 \pm 18.2$ & $85.7 \pm 14.3$ & $52.4 \pm 9.5$ \\
\hline 32 & $50^{+}$ & 100 & 56.7 & 100 & 43.3 & 100 & 43.3 \\
\hline 33 & $50^{+}$ & 100 & 45.8 & 75.0 & 12.5 & 12.5 & 4.2 \\
\hline
\end{tabular}




\begin{tabular}{|c|c|c|c|c|c|c|c|}
\hline \multirow{2}{*}{$\begin{array}{l}\text { Compound } \\
\text { (No.) }\end{array}$} & \multirow{2}{*}{$\begin{array}{l}\text { Concentration } \\
(\mu \mathrm{M})\end{array}$} & \multicolumn{2}{|c|}{$24 \mathrm{~h}$} & \multicolumn{2}{|c|}{$48 \mathrm{~h}$} & \multicolumn{2}{|c|}{$72 \mathrm{~h}$} \\
\hline & & $\begin{array}{l}\text { Mobile } \\
\text { worm (\%) }\end{array}$ & $\begin{array}{l}\text { Mobility } \\
\text { score (\%) }\end{array}$ & $\begin{array}{l}\text { Mobile } \\
\text { worm (\%) }\end{array}$ & $\begin{array}{l}\text { Mobility } \\
\text { score (\%) }\end{array}$ & $\begin{array}{l}\text { Mobile } \\
\text { worm (\%) }\end{array}$ & $\begin{array}{l}\text { Mobility } \\
\text { score (\%) }\end{array}$ \\
\hline 34 & $50^{+}$ & 100 & 38.1 & 100 & 38.1 & 100 & 38.1 \\
\hline \multirow[t]{3}{*}{36} & $50^{+}$ & 100 & 83.3 & 50.0 & 16.7 & 0 & 0 \\
\hline & 25 & 100 & $93.3 \pm 1.2$ & 100 & $66.7 \pm 15.8$ & $10.0 \pm 20.0$ & $3.3 \pm 16.7$ \\
\hline & 10 & 100 & $84.8 \pm 5.6$ & 100 & $56.0 \pm 26.0$ & 100 & $\begin{array}{l}45.5 \\
\pm 13.6\end{array}$ \\
\hline 37 & $50^{+}$ & 100 & 70.8 & 93.8 & 56.3 & 87.5 & 52.1 \\
\hline \multirow[t]{2}{*}{38} & $50^{+}$ & 21.4 & 7.1 & 0 & 0 & 0 & 0 \\
\hline & 25 & 100 & $98.6 \pm 0.2$ & 100 & $85.5 \pm 3.0$ & $65.2 \pm 13.0$ & $55.1 \pm 8.7$ \\
\hline \multirow[t]{3}{*}{39} & $50^{+}$ & 80.0 & 26.7 & 60.0 & 12.0 & 0 & 0 \\
\hline & 25 & $66.7 \pm 22.3$ & $29.6 \pm 32.0$ & $55.6 \pm 28.0$ & $25.9 \pm 32.5$ & $33.3 \pm 22.2$ & $11.1 \pm 11.1$ \\
\hline & 10 & 100 & $33.3 \pm 12.6$ & 100 & $33.3 \pm 12.6$ & $85.7 \pm 14.2$ & $42.9 \pm 9.5$ \\
\hline \multirow[t]{3}{*}{40} & $50^{+}$ & 20.0 & 6.7 & 0 & 0 & 0 & 0 \\
\hline & 25 & 100 & $66.7 \pm 11.1$ & $85.7 \pm 4.3$ & $38.1 \pm 11.6$ & $42.9 \pm 28.6$ & $19.0 \pm 19.0$ \\
\hline & 10 & 100 & $66.7 \pm 33.3$ & 100 & $66.7 \pm 33.3$ & 100 & $\begin{array}{l}33.3 \\
\pm 20.0\end{array}$ \\
\hline \multirow[t]{3}{*}{41} & $50^{+}$ & 33.3 & 11.1 & 0 & 0 & 0 & 0 \\
\hline & 25 & 100 & $33.3 \pm 14.3$ & $92.3 \pm 5.1$ & $30.8 \pm 23.8$ & $53.8 \pm 15.4$ & $17.9 \pm 10.3$ \\
\hline & 10 & 100 & $37.5 \pm 12.5$ & 100 & $37.5 \pm 12.5$ & 100 & $37.5 \pm 12.5$ \\
\hline 42 & $50^{+}$ & 100 & 66.7 & 90.0 & 60.0 & 70.0 & 26.7 \\
\hline 43 & $50^{+}$ & 100 & 66.7 & 100 & 66.7 & 100 & 66.7 \\
\hline \multirow[t]{3}{*}{44} & $50^{+}$ & 14.2 & 4.8 & 0 & 0 & 0 & 0 \\
\hline & 25 & 100 & $33.3 \pm 17.7$ & $10.0 \pm 5.0$ & $3.3 \pm 3.3$ & 0 & 0 \\
\hline & 10 & 100 & $83.3 \pm 6.7$ & 100 & $66.7 \pm 15.7$ & $93.8 \pm 12.5$ & $47.9 \pm 8.3$ \\
\hline 45 & $50^{+}$ & 100 & 51.9 & 100 & 51.9 & 100 & 51.9 \\
\hline \multirow[t]{2}{*}{ PZQ } & 50 & 100 & $33.3 \pm 0.0$ & 100 & $33.3 \pm 0.0$ & 100 & $33.3 \pm 0.0$ \\
\hline & 25 & 100 & $33.3 \pm 0.0$ & 100 & $33.3 \pm 0.0$ & 100 & $33.3 \pm 0.0$ \\
\hline Art & 50 & 100 & 100 & 100 & 100 & 100 & 100 \\
\hline $\begin{array}{l}\text { `Experiments we } \\
\text { The most efficie } \\
6-8 \text { worms wer } \\
\text { Art: Artesunate; }\end{array}$ & $\begin{array}{l}\text { ere performed once. } \\
\text { ent compounds are in } \\
\text { e used. } \\
\text {; PZQ: Praziquante. }\end{array}$ & $\begin{array}{l}\text { The others were } \\
\text { bold font. See st }\end{array}$ & $\begin{array}{l}\text { e three times } \\
\text { orting inform }\end{array}$ & $\begin{array}{l}\text { average } \pm S D \\
\text { for mobile } W\end{array}$ & $\begin{array}{l}\text { iven. } \\
\text { רand mobility s }\end{array}$ & e definitions. Fo & each group, \\
\hline
\end{tabular}

to achieve higher worm killing efficacy. Meanwhile, the in vivo metabolic stability or pharmacokinetic parameters of the compounds should be further optimized.

\section{Conclusion}

In summary, we have designed and prepared a series of endoperoxide and PZQ conjugates. The in vitro activity of these compounds against both adult and juvenile $S$.

\section{Key terms:}

Peroxide: Compound containing the oxygen-oxygen single bond. japonicum, and against S. mansoni as well, was thoroughly investigated. In general, these compounds exhibited consistent activity to both species of worms. Although some conjugates exhibited high efficacy at low concentration, the promising in vitro efficacy leads to significant but moderate worm burden reduction activity in vivo. Nevertheless, this research provided important insights into antischistosomal agents which executed the hybrid drug design rationale.

\section{Future perspective}

Schistosomiasis is listed as one of the 17th NTDs. Since the 1970 s, praziquantel has been the only drug 
Table 3. Effects of the compounds at $50 \mu \mathrm{M}$ on adult Schistosoma mansoni in vitro.

\begin{tabular}{|c|c|c|c|c|c|c|}
\hline \multirow{2}{*}{$\begin{array}{l}\text { Compound } \\
\text { (No.) }\end{array}$} & \multicolumn{2}{|c|}{$24 \mathrm{~h}$} & \multicolumn{2}{|c|}{$48 h$} & \multicolumn{2}{|c|}{$72 \mathrm{~h}$} \\
\hline & $\begin{array}{l}\text { Mobile worm } \\
(\%)\end{array}$ & $\begin{array}{l}\text { Mobility score } \\
(\%)\end{array}$ & $\begin{array}{l}\text { Mobile } \\
\text { worm (\%) }\end{array}$ & $\begin{array}{l}\text { Mobility } \\
\text { score (\%) }\end{array}$ & $\begin{array}{l}\text { Mobile } \\
\text { worm (\%) }\end{array}$ & $\begin{array}{l}\text { Mobility } \\
\text { score (\%) }\end{array}$ \\
\hline 7 & $93.8 \pm 6$ & $53.1 \pm 9$ & $12.5 \pm 13$ & $6.3 \pm 6$ & 0 & 0 \\
\hline 8 & 0 & 0 & 0 & 0 & 0 & 0 \\
\hline 9 & $6.3 \pm 6$ & $3.1 \pm 3$ & 0 & 0 & 0 & 0 \\
\hline 10 & 0 & 0 & 0 & 0 & 0 & 0 \\
\hline 11 & $12.5 \pm 13$ & $6.3 \pm 6$ & 0 & 0 & 0 & 0 \\
\hline 12 & 0 & 0 & 0 & 0 & 0 & 0 \\
\hline 13 & 100 & $59.4 \pm 3$ & $75.0 \pm 25$ & $37.5 \pm 13$ & 43.86 & $21.9 \pm 3$ \\
\hline 14 & 100 & $71.9 \pm 9$ & $87.5 \pm 13$ & $56.3 \pm 6$ & $68.8 \pm 6$ & $46.9 \pm 9$ \\
\hline 15 & 100 & $90.6 \pm 9$ & 100 & $87.5 \pm 6$ & 100 & $87.5 \pm 6$ \\
\hline 16 & 100 & 100 & 100 & 100 & $93.8 \pm 6$ & $93.8 \pm 6$ \\
\hline 17 & 100 & $84.4 \pm 9$ & 0 & 0 & 0 & 0 \\
\hline 18 & 100 & 100 & 100 & $68.8 \pm 6$ & $62.5 \pm 13$ & $31.3 \pm 6$ \\
\hline 19 & 100 & 100 & $25 \pm 13$ & $12.5 \pm 6$ & 0 & 0 \\
\hline 20 & 100 & 100 & $50 \pm 0$ & $25 \pm 0$ & $18.8 \pm 6$ & $9.4 \pm 3$ \\
\hline 29 & 100 & $71.9 \pm 3$ & $25.0 \pm 0$ & $12.5 \pm 0$ & $12.5 \pm 13$ & $6.3 \pm 6$ \\
\hline 30 & 100 & $87.5 \pm 13$ & $18.8 \pm 19$ & $9.4 \pm 9$ & 0 & 0 \\
\hline 31 & $37.5 \pm 13$ & $18.8 \pm 6$ & 0 & 0 & 0 & 0 \\
\hline 32 & 100 & $71.9 \pm 9$ & $31.3 \pm 6$ & $15.6 \pm 3$ & $25 \pm 13$ & $12.5 \pm 6$ \\
\hline 33 & 100 & $59.4 \pm 9$ & $12.5 \pm 13$ & $6.3 \pm 6$ & 0 & 0 \\
\hline 34 & $12.5 \pm 13$ & $6.3 \pm 6$ & 0 & 0 & 0 & 0 \\
\hline 36 & $87.5 \pm 0$ & $62.5 \pm 6$ & $68.8 \pm 19$ & $43.8 \pm 13$ & $12.5 \pm 13$ & $6.3 \pm 6$ \\
\hline 37 & 100 & 100 & $87.5 \pm 13$ & $75 \pm 19$ & $75 \pm 19$ & $50 \pm 0$ \\
\hline 38 & $31.3 \pm 6$ & $15.6 \pm 3$ & $50 \pm 13$ & $25 \pm 6$ & $37.5 \pm 0$ & $18.8 \pm 0$ \\
\hline 39 & 100 & 100 & 100 & $90.6 \pm 3$ & 100 & $87.5 \pm 0$ \\
\hline 40 & 100 & $87.5 \pm 13$ & $68.8 \pm 6$ & $40.6 \pm 3$ & $25.0 \pm 13$ & $12.5 \pm 6$ \\
\hline 41 & 100 & 100 & 100 & $84.4 \pm 3$ & 100 & $71.9 \pm 3$ \\
\hline 42 & $87.5 \pm 13$ & $43.8 \pm 6$ & $81.3 \pm 19$ & $40.6 \pm 19$ & $75 \pm 13$ & $37.5 \pm 6$ \\
\hline 43 & 100 & 100 & $37.5 \pm 0$ & $21.9 \pm 3$ & $25 \pm 0$ & $12.5 \pm 0$ \\
\hline 44 & $50.0 \pm 13$ & $28.1 \pm 3$ & $37.5 \pm 0$ & $18.8 \pm 0$ & $6.3 \pm 6$ & $3.1 \pm 3$ \\
\hline 45 & $62.5 \pm 38$ & $37.5 \pm 25$ & 0 & 0 & 0 & 0 \\
\hline Praziquante & 0 & 0 & 0 & 0 & 0 & 0 \\
\hline Control & 100 & 100 & 100 & 100 & 100 & 100 \\
\hline
\end{tabular}

of choice for the treatment of this disease. Recently, Schistosoma with low sensitivity or resistance to praziquantel have been observed and reported. Therefore, there is an urgent need for the discovery of new and effective antischistosomal drugs.

The present study reported the design, synthesis and evaluation a series of synthetic peroxide and praziquantel conjugates with high worm inhibition activity against both $S$. japonicum and $S$. mansoni. Although highly efficient in vitro, these compounds did not display promising worm killing ability in vivo. This research provided insights into antischistosomal drug discovery using the hybrid drug design rationale. Consequently, efforts to search for new 


\begin{tabular}{|c|c|c|}
\hline Compounds & Number of detected worms/mice (mean \pm SD) & Worm burden reduction rate (\%) \\
\hline Control & $43 \pm 3.9$ & 0 \\
\hline 9 & $41 \pm 1.7$ & 3.9 \\
\hline 11 & $32 \pm 3.5$ & $25.1^{* *}$ \\
\hline 15 & $30 \pm 4.9$ & $29.3^{* *}$ \\
\hline 16 & $39 \pm 4.0$ & 10.0 \\
\hline 17 & $29 \pm 1.6$ & $32.2^{* *}$ \\
\hline 19 & $37 \pm 2.8$ & $14.7^{*}$ \\
\hline 31 & $31 \pm 3.0$ & $28.6^{* *}$ \\
\hline 44 & $37 \pm 3.9$ & $14.4^{*}$ \\
\hline Praziquante & $14 \pm 3.9$ & $67.7^{\star *}$ \\
\hline
\end{tabular}

\begin{tabular}{l} 
Table 5. Effect of single $400 \mathrm{mg} / \mathrm{kg}$ oral dose of the compounds on mice harboring 14 day-old \\
juvenile Schistosoma japonicum. \\
\begin{tabular}{|lll|}
\hline Compounds & Number of detected worms/mice (mean \pm SD) & Worm burden reduction rate (\%) \\
Control & $39 \pm 5.9$ & 0 \\
\hline 7 & $23 \pm 4.4$ & $42.8^{* *}$ \\
9 & $26 \pm 2.3$ & $35.2^{* *}$ \\
\hline 11 & $31 \pm 3.9$ & $21.5^{*}$ \\
\hline 17 & $32 \pm 3.6$ & 20.2 \\
\hline 19 & $36 \pm 1.1$ & 9.4 \\
\hline 44 & $39 \pm 3.6$ & 0 \\
\hline Artesunate & $10 \pm 0.8$ & $74.7^{* *}$ \\
\hline Five mice were used in each group. T-test was used to compare each compounds with the control group $\left({ }^{*} p<0.05, * * p<0.01\right)$.
\end{tabular} \\
\hline
\end{tabular}

hybrid molecules as antischistosomal agents need to be considered. Meanwhile, further studies concerning the compound pharmacokinetic property as well as bioavailability should be pursued and combined in the following research.

Acknowledgments

We thank Zhongwu Guo from Wayne State University for the language editing.
Financial \& competing interests disclosure

This project is funded by the Priority Academic Program Development of Jiangsu Higher Education Institutions. The authors have no other relevant affiliations or financial involvement with any organization or entity with a financial interest in or financial conflict with the subject matter or materials discussed in the manuscript apart from those disclosed.

No writing assistance was utilized in the production of this manuscript.

\section{Executive summary}

- Praziquantel and synthetic peroxide conjugates displayed high worm killing activity against both juvenile and adult Schistosoma.

- The type of the linker and the peroxide moiety in the conjugates play a significant role for their antischistosomal activity. Compounds with amide linker and 1,2,4,5-tetraoxane as pharmacophore exhibited higher inhibitory activity.

- These conjugates exhibited consistent worm killing profile against Schistosoma japonicum and Schistosoma mansoni.

- Although highly efficient in vitro, these conjugates show low to moderate worm reducing activity in vivo. 


\section{References}

Papers of special note have been highlighted as:

- of interest; $\bullet \bullet$ of considerable interest

1 Njoroge M, Njuguna NM, Mutai P et al. Recent Approaches to chemical discovery and development against malaria and the neglected tropical diseases human African trypanosomiasis and schistosomiasis. Chem. Rev. 114, 11138-11163 (2014).

- Very interesting review regarding drug discovery in the field of neglected tropical disease.

2 Cioli D, Pica-Mattoccia L. Praziquantel. Parasitol. Res. 90(1), S3-S9 (2003).

3 King $\mathrm{CH}$, Mahmoud AAF. Drugs five years later: praziquantel. Annals 110(4), 290-296 (1989).

4 Ismail M, Botros S, Metwally A et al. Resistance to praziquantel: direct evidence from Schistosoma mansoni isolated from Egyptian villagers. Am. J. Trop. Med. Hyg. 60, 932-935 (1999).

5 Gryseels B, Mbaye A, de Vlas SJ et al. Are poor responses to praziquantel for the treatment of Schistosomamansoni infections in Senegal due to resistance? An overview of the evidence. Trop. Med. Int. Health 6, 864-873 (2001).

6 Doenhoff MJ, Cioli D, Utzinger J. Praziquantel: mechanism of action, resistance and new derivatives for schistosomiasis. Curr. Opin. Infect. Dis. 21, 659-667 (2008).

-. Very interesting review regarding the anti-schistosomal drug praziquantel.

7 Nepali K, Sharma S, Sharma M et al. Rational approaches, design strategies, structure activity relationship and mechanistic insights for anticancer hybrids, Eur. J. Med. Chem. 77, 422-487 (2014).

- Very interesting review about hybrid drug design rationale.

8 Laurent SA, Boissier J, Cosledan F et al. Synthesis of "Trioxaquantel" derivatives as potent new antischistosomal drugs. Eur. J. Org. Chem. 10, 895-913 (2008).

- Very interesting research article about anti-schistosomal agents

9 Dechy-Cabaret O, Benoit-Vical F, Robert A et al. Preparation and antimalarial activities of "Trioxaquines", new modular molecules with a trioxane skeleton linked to a 4-aminoquinoline. ChemBioChem. 1, 281-283 (2000).
10 Portela J, Boissier J, Gourbal B et al. Antischistosomal activity of Trioxaquines: in vivo efficacy and mechanism of action on schistosoma mansoni. PLoS Negl. Trop. Dis. 6, e1474 (2012).

- An interesting research article about hybrid drug trioxaquines as anti-schistosomal agents.

11 Dong Y, Chollet J, Vargas M et al. Praziquantel analogs with activity against juvenile Schistosoma mansoni. Bioorg. Med. Chem. Lett. 20, 2481-2484 (2010).

- An interesting research article about praziquantel derivatives as anti-schistosomal agents.

12 Patra M, Ingram K, Pierroz V et al. Ferrocenyl derivatives of the anthelmintic praziquantel: design, synthesis, and biological evaluation. J. Med. Chem. 55, 8790-8798 (2012).

13 Patra M, Ingram K, Leonidova A. In vitro metabolic profile and in vivo antischistosomal activity studies of $\left(\eta^{6}\right.$-Praziquantel $) \mathrm{Cr}(\mathrm{CO})_{3}$ derivatives. J. Med. Chem. 56, 9192-9198 (2013).

14 Duan W, Qiu S, Zhao Y et al. Praziquantel derivatives exhibit activity against both juvenile and adult Schistosomajaponicum. Bioorg. Med. Chem. Lett. 22, 1587-1590 (2012).

15 Dong L, Duan W, Chen J. An artemisinin derivative of praziquantel as an orally active antischistosomal agent. PLoS ONE 9, e116123 (2014).

-• Published work by us, related to this research.

16 Charman A A, Arbe-Baunes I C, Brun R et al. Synthetic ozonide drug candidate OZ 439 offers new hope for a singledose cure of uncomplicated malaria. Proc. Natl Acad. Sci. USA 108, 4400-4405 (2011).

17 Yan X, Chen J, Zhu Y-T et al. Phosphomolybdic acid catalyzed synthesis of 1,2,4,5-tetraoxane. SYNLETT 19, 2828-2830 (2011).

18 Amewu R K, Chadwick J, Hussain A. Synthesis and evaluation of the antimalarial, anticancer, and caspase 3 activities of tetraoxane dimers. Bioorg. Med. Chem. 21, 7392-7397 (2013).

19 Zhao Q, Vargas M, Dong Y. Structure-activity relationship of an ozonidecarboxylic acid (OZ78) against Fasciola hepatica. J. Med. Chem. 53, 4223-4233 (2010).

20 Xiao S-H, Keise J, Chollet J et al. In vitro and in vivo activities of synthetic trioxolanes against major human schistosome species. Antimicrob. Agents Chemother. 51(4), 1440-1445 (2007). 